\title{
Investigating Ischemic Stroke Costs and Filling a Critical Knowledge Gap
}

Can J Neurol Sci. 2012; 39: 697

Stroke is one of the most significant health problems in North America. Stroke is ranked amongst the most expensive chronic diseases along with cancer, diabetes, and depression. ${ }^{1-3}$ Stroke imposes a significant economic burden on our healthcare system, with acute ischemic stroke accounting for the bulk of costs. Direct costs encompass all expenditures from hospitalization, nursing homes, physicians, and other healthcare professionals, drugs, other medical durables, and home healthcare, whereas indirect costs accounts for lost productivity due to morbidity and mortality.

Despite the high financial burden, limited recent research investigations have focused on costs associated with stroke in Canada and the United States.

A systematic literature review examining the cost burden of ischemic stroke was conducted to determine if a gap in available information existed. ${ }^{4}$ The search yielded 417 potential studies which were screened for eligibility, but ultimately only 28 articles met inclusion criteria. Most cost-analysis studies conducted thus far have focused on short-term, in-hospital costs of stroke. Few studies examined long-term (beyond 30 days post stroke) direct costs. Those that did were largely retrospective analyses. The systematic review also uncovered the relative scarcity of quality studies focusing on the short and longer term indirect costs of stroke, which are substantial. Finally, almost all the studies citing short and long-term costs of stroke were conducted in the 1990's, highlighting the need for current era data on the costs of stroke.

The knowledge gap has been filled. Finally, we have high quality, valid, prospective, longitudinal, patient-level data on resource use and direct and indirect, short and long-term costs after ischemic stroke. ${ }^{5}$

Mittmann et al enrolled 232 patients with ischemic stroke from 12 Canadian stroke centers into the Economic Burden of Ischemic Stroke (BURST) Study, followed the cohort prospectively, collected clinical history, disability, health preference, and resource utilization (both direct and indirect costs) out to one year, and stratified by disability measured at discharge using modified Rankin Score $(\mathrm{mRS}) .^{5}$ The average annual cost was $\$ 74,353$; $\$ 48,339$ for non-disabling (mRS 0-2) and more than double $(\$ 107,883)$ for disabling strokes (mRS 3-5). Equally important, the authors determined how the costs varied during each successive phase of stroke care. Costs during the hospitalization to three months phase were the highest contributor to the annual cost. Similar to US analyses, hospitalization and rehabilitation costs, combined, represented $80.8 \%$ of the total average cost for this time period. ${ }^{6}$ Mittmann et al reported that during the four to six months phase and last phase, indirect costs $(58.3 \%$ and $42.3 \%)$ were a major cost driver, consistent with Taylor et al who reported that indirect costs accounted for $58 \%$ of lifetime stroke costs in the United States. 5,7
Mittmann et al novel study demonstrated how stroke related disability and phase of stroke care both impact costs. ${ }^{5}$ The authors recognize that costs by severity of disease are critical to decision makers responsible for evaluating new therapies. It stands to reason that direct and indirect costs associated with stroke can be reduced by broad adoption of new and existing strategies for ischemic stroke care. Designing future cost effectiveness analyses will be much easier with this critical stroke cost knowledge cap already filled.

\section{Bart M. Demaerschalk \\ Mayo Clinic, Phoenix, Arizona, USA}

\section{REFERENCES}

1. American Cancer Society. Cancer facts \& figures 2008. Atlanta, GA: America Cancer Society, Inc; 2008.

2. American Diabetes Association. Economic costs of diabetes in the US in 2007. Diabetes Care. 2008;31(3):596-615.

3. Greenberg PE, Kessler RC, Birnbaum HG, et al. The economic burden of depression in the United States; How did it change between 1990 and 2000? J Clin Psychiatry. 2003;64(12): 1465-75.

4. Demaerschalk BM, Hwang Ha-Mill, Leung G. US cost burden of ischemic stroke: a systematic literature review. Am J Manag Care. 2010;16(7):525-33.

5. Mittmann N, Seung SJ, Hill MD, et al. Impact of disability status on ischemic stroke costs in Canada in the first year. Can J Neurol Sci. 2012;39(6):793-800.

6. Lee WC, Christensen MC, Joshi AV, Pashos CL. Long-term cost of stroke subtypes among Medicare beneficiaries. Cerebrovasc Dis. 2007;23(1):57-65.

7. Taylor TN, David PH, Torner JC, Holmes J, Meyer JW, Jacobson MF. Lifetime cost of stroke in the United States. Stroke. 1996; 27(9):1459-66. 